\title{
PROSPECTIVA DEL MERCADO DE ACTIVOS AMBIENTALES EN EL PERÚ
}

\author{
Elsa Esther Choy Zevallos* \\ Docente Asociada de la Facultad de Ciencias Contables \\ Universidad Nacional Mayor de San Marcos-UNMSM / Lima-Perú \\ [Recepción: Mayo de 2013/Conformidad: Junio de 2013]
}

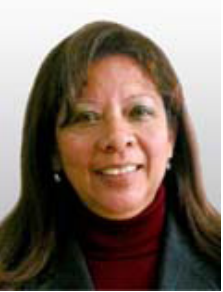

\section{RESUMEN}

El desconocimiento del valor económico de los recursos naturales ha contribuido a su deterioro $y$ degradación, que afecta la situación económica y social de nuestro país, la razón es que existe un mercado definido para las transacciones.

La valoración de los recursos naturales como objetivo del desarrollo sostenible, propone que el medio ambiente no sea un bien libre, aunque no existan mercados convencionales, sugiere medir su utilización y escasez. Debe incluir el valor económico total del recurso natural incluye valores: directos, indirectos, presentes, futuros, de opción, de uso, no uso, y de existencia.

Es previsible que progresivamente se configure un mercado real de activos ambientales, es necesario tener parámetros de valorización, que sirvan para generar alternativas de desarrollo en comunidades rurales, manteniendo prácticas no contaminantes. El objetivo es identificar los recursos potenciales y formalmente transables, que permita información de base para aplicar una metodología de valorización, construyendo un escenario futuro de mercado de activos ambientales.

Palabras clave: mercado activos ambientales, recursos naturales, métodos valorización

\begin{abstract}
The ignorance of the natural resources economic has contributed to its deterioration and degradation, which affects the social and economic situation of our country, the reason is that there is a distinct market for transactions.

The valuation of natural resources is sustainable trough an objective development, proposed that the environment is not a free, although there are no conventional markets, and suggests measuring use and shortage. It must be included the total economic value of the natural resource values which are: direct, indirect, present, future, option, of use, not used, and existence. It is foresee gradually the set up a real market of environmental assets, it is necessary have parameter of valuation, that would serve to generate alternative developments in rural communities, keeping clean environmental practices.

The objective is to identify formally tradable and potential resources that allows information which are going to be apply for a methodic valorization, that would allow us to build a future scenario of environmental assets in the market.
\end{abstract}

Keywords: market environmental assets, natural resources, valorization methodic

\footnotetext{
Doctora en Ciencias Contables y Empresariales. Magíster en Dirección Financiera. Contadora Pública Colegiada Certifica. Docente Investigadora-UNMSM.E-mail: eschoyz@yahoo.com
} 


\section{ANTECEDENTES}

En el Perú, desde hace más de una década existen estudios que evalúan los proyectos ambientales, asimismo, diversas organizaciones promueven proyectos de activos ambientales, una de ellas es VSF CICDA-PERÚ, proyecto que está en la sierra de Piura, en la parte alta, se ejecuta con comunidades campesinas que están sembrando bosques para la captura de carbono. Se logró captar el interés de la empresa inglesa Twin Trading, compradora de café en el Perú. El proyecto se ubica en el distrito de Yamango, de la provincia de Morropón, en comunidad de Choco, a 3500 metros sobre el nivel del mar e involucra a 15 pequeños caseríos. Es decir, cerca de mil familias son las que están reforestando estas zonas con especies nativas, de capulíes de aliso y con la siembra de pino. El proceso es el siguiente: el bióxido de carbono que está en el ambiente se captura mediante los árboles, a través de la fotosíntesis y esto después se cuantifica. La cuantificación se realiza mediante un proceso de certificación y es esta la que se comercializa en el mercado internacional.

El mercado voluntario de carbono fue materia del CADE de Ejecutivos 2012, donde se menciona el desarrollo de un incipiente mercado de activos ambientales, comenzando con el "mercado voluntario de carbono", el cual según el informe "Developing Dimension: State of the Voluntary Carbon Markets 2012" elaborado por Ecosystem Marketplace y Bloomberg New Energy Finance, en el año 2011 ha registrado transacciones por US\$ 576 millones y las $95 \mathrm{MtCO} 2 \mathrm{e}$, registrándose un incremento del 35\% en relación al año 2010, a pesar de la crisis financiera internacional, observándose que los compradores europeos incrementaron sus operaciones y los compradores norteamericanos y aquellos provenientes de mercados emergentes hicieron su entrada

En la tendencia mundial es cada vez mayor la preocupación por preservar el ambiente; dado que toda actividad humana, económica o no, genera efectos sobre las condiciones que rodean la vida humana. En ese sentido, las empresas buscan reducir los efectos contaminantes de sus actividades corrientes y antes de iniciar sus proyectos, estudian sus impactos.
Organismos internacionales como ONU, FAO, o el Banco Mundial se encargan de motivar proyectos ambientales, que contablemente significan activos apreciables y rentables en las empresas.

\section{LOS BIENES AMBIENTALES}

Los bienes ambientales, por lo general, no cuentan con un mecanismo de valorización para determinar su precio, cuando esto ocurre, como es el caso de algunos recursos naturales como el agua, petróleo, este precio es solo una valoración a corto plazo y que por lo tanto no toma en cuenta el agotamiento que inexorablemente ocurrirá a mediano o largo plazo si se continua con el nivel de utilización.

Si la mayoría de industrias utiliza el recurso natural agua para el proceso de transformación, surgiría entonces la interrogante iel mercado de bienes y servicios refleja el verdadero valor de los recursos que han sido empleados para su producción? Si la respuesta es negativa, significaría que el mercado no distribuye de manera eficiente los recursos al no contemplar el valor de un recurso natural no renovable como es el agua u otro que cumple un rol importante en el medio ambiente. Algunos autores consideran fallos de mercado y da lugar a que los costos externos en los que se incurren en el proceso de producción no queden reflejados en los precios finales, surgiendo así el concepto de externalidades.

Según Santos M. Ruesga y Gemma Durán, Empresa y Medio Ambiente (1995), se afirma que existe una externalidad cuando la producción o el consumo de un bien afecta directamente a los consumidores o empresas que no participan en su compra ni en su venta, y cuando los efectos no se reflejan totalmente en los precios de mercado. Se dice que existen externalidades positivas cuando obtenemos una ventaja o beneficio por lo que otros hacen, mientras que si es un perjuicio hablamos de externalidades negativas.

Una de las razones por la cual los bienes ambientales no son valorados adecuadamente se debe a que no existe un mercado definido para la transacción de los mismos, por tratarse de recursos que pueden obtenerse libremente, considerando que los recur-

8/ QVIPURAMAYOC | Vol. 21(39) 2013 
sos ambientales no son de propiedad privada. Son denominados recursos de propiedad común por el hecho de que se tiene libre acceso a ellos. Es este libre acceso, precisamente, la causa del abuso en su uso, ya que cuando se utilizan como factores de producción, poseen un coste nulo o muy por debajo de su "costo social" si se tomaran en cuenta los efectos negativos ambientales, tales como, agotamiento, contaminación, desastres ecológicos, entre otros.

\section{VALOR DE LOS ACTIVOS AMBIENTALES}

Existe diferencia entre bienes y servicios económicos y no económicos. Los primeros son aquellos que alcanzan un precio, expresado en unidades monetarias, al ser intercambiados en el mercado y es el mercado el que regula su escasez o abundancia relativa. En cambio, el valor de los no económicos, en particular los bienes ambientales, considerados libres, es poco reconocido y difícilmente es homogéneo a la unidad que los bienes económicos, por tanto el mercado encuentra dificultades para determinar un precio que regule su utilización.

Sin embargo, la valoración de los recursos naturales es uno de los objetivos del desarrollo sostenible. Este concepto propone que el medio ambiente no sea un bien libre, aunque no existan mercados convencionales para los mismos, y sugiere que se mida utilizando una metodología adecuada.

Una valoración adecuada tendría que incorporar el valor económico total de un recurso natural que incluya no solo los valores directos e indirectos presentes, sino también futuros, valor de opción, derivados de su valor de uso y de su valor de no uso, es decir, el valor de existencia de los recursos naturales.

Tal como se observa en el siguiente gráfico:

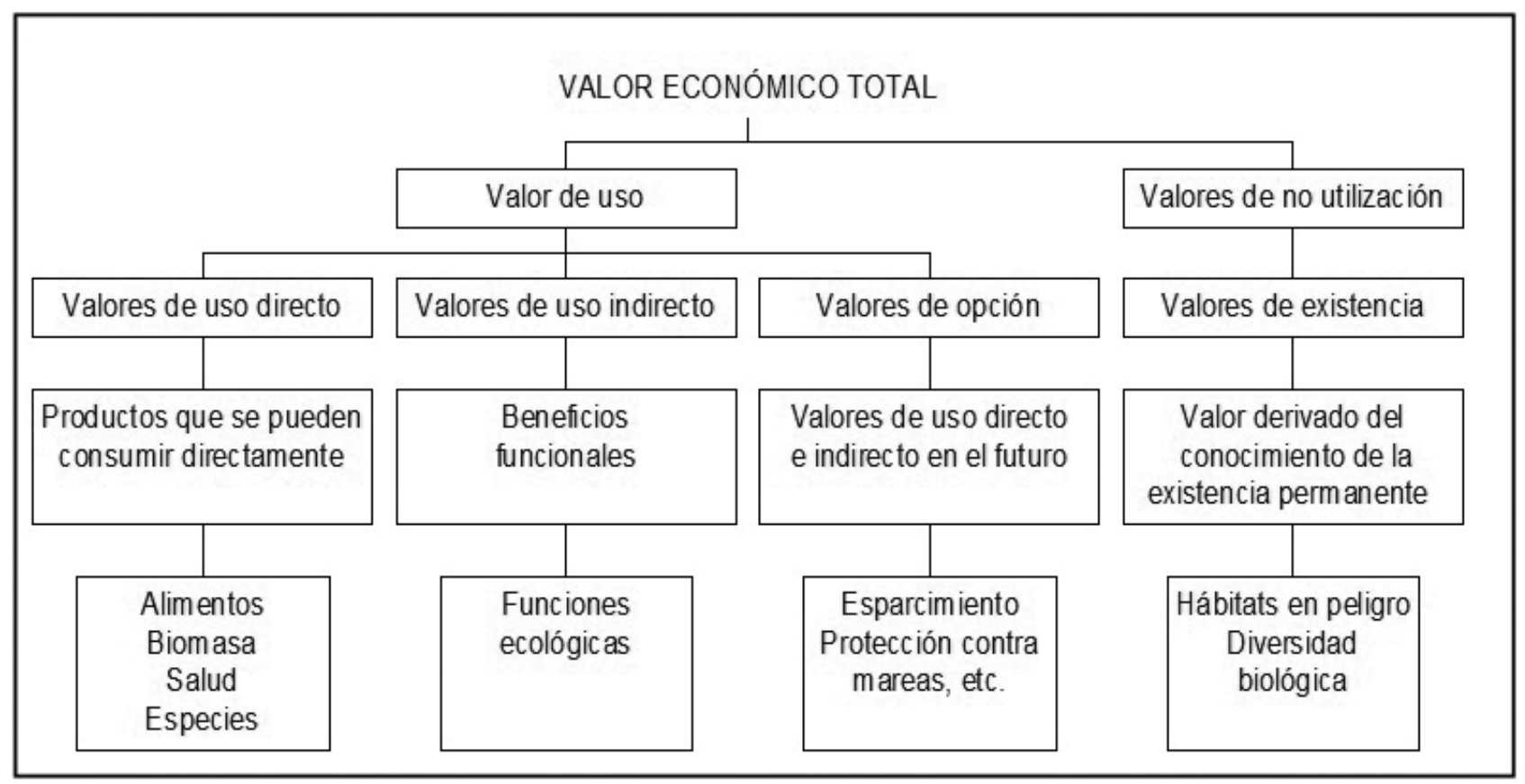

Gráfico 1. Santos M. Ruesga y Gemma Durán. Empresa y Medio Ambiente. 1995.

El valor de uso es aquel que se atribuye por parte de las personas que usufructúan el medio ambiente, el valor de opción está relacionado con la posibilidad de uso futuro, y por último, el valor de existencia representa un valor atribuido directamente por la subsistencia del medio ambiente independiente de un uso presente o futuro.
La valoración monetaria de los bienes ambientales permitiría su incorporación en la función de producción de las empresas, lo que llevaría a una necesaria e ineludible consideración de los costos ambientales a la hora de obtener un producto o servicio, contribuyendo de esta forma con los objetivos del desarrollo sostenible. 


\section{MERCADO DE ACTIVOS AMBIENTALES EN EL PERÚ}

En nuestro país el mercado de activos ambientales son materia relativamente reciente en investigación. La abundancia de nuestros recursos naturales y el poco entendimiento de las propiedades y funciones de los ecosistemas no han permitido desarrollar un sistema de precios de mercado. Sin embargo, bajo el concepto de desarrollo sostenible emerge la necesidad de valorar estos recursos naturales que han sido excluidos en la toma de decisiones políticas

Si bien se cuenta con avances metodológicos en la valorización económica de los activos ambientales, son pocos los estudios que profundizan a este nivel de investigación, lo cual podría atribuirse a la complejidad en las metodologías sobre valoración económica ambiental o la controversia que todavía estas suscitan.

El sector académico juega un papel prioritario desde los centros de educación superior como la Universidad Nacional Mayor de San Marcos, desde donde de manera directa o indirectamente y gracias a las políticas y estrategias, la investigación e innovación, y los procesos de capacitación que se desarrollan de forma integrada y multidisciplinaria, generan un gran marco de conocimiento que potencia el desempeño ambiental.

\section{CONCLUSIONES}

El uso indiscriminado de tecnología por aquellas empresas que no cumplen con la legislación ambiental, es una amenaza para la población y atenta contra la vida y salud de la persona, un derecho constitucional que no debe ser afectado a consecuencia del deterioro ambiental.

En el Perú no existe un mercado de activos ambientales, una de las razones se debe a las dificultades en la valoración del precio de recursos naturales al no utilizar una metodología adecuada.

Existen bienes sacrificados en el proceso de producción, denominados recursos naturales, los cuales por carecer de un componente monetario no quedan reflejados en el costo de producción de la empresa.

\section{RECOMENDACIONES}

El Estado debe cumplir su rol con el cuidado y preservación del ambiente, aplicando los controles y legislación rigurosa para aquellas industrias que afectan el medio ambiente, induciendo a utilizar tecnología limpia, con materiales menos contaminantes y energía renovable.

Los agentes económicos deben preocuparse en aplicar una metodología adecuada a fin de determinar un componente monetario para los recursos naturales, de tal forma que el concepto de "valor" para bienes ambientales que solían ser clasificados, desde el punto de vista económico, como bienes "libres", lograría una utilización más eficiente de los mismos, lo que redundaría en beneficios para la sociedad en su conjunto.

La teoría de los costos no solo debe estar presente en los procesos de producción de bienes y servicios, sino también en una adecuada valorización de los recursos naturales consumidos.

\section{REFERENCIAS BIBLIOGRÁFICAS}

1. Arias, G. (2004). Análisis del impacto económico y social de las plantaciones forestales en Costa Rica. FUNDECOR. San José. Costa Rica

2. Barrantes, J (2005). Programa de Pago de Servicios Ambientales PSA, su evolución entre 19972004. ONF. México.

3. De Camino, R. (2005). El manejo de bosques y plantaciones forestales al 2025 en Costa Rica. ¿Qué podemos esperar? El sector forestal en Costa Rica, perspectivas al 2025. Costa Rica.

4. Ruesga , Sy Durán, G. (1995). Empresa y Medio Ambiente.

5. Quintero, M. (2006). Modelo de Optimización para Evaluación Ex Ante de Alternativas Productivas y Cuantificación de Externalidades Ambientales en Cuencas Andinas. Proyecto Regional Cuencas Andinas CONDESAN y Centro Internacional de Agricultura Tropical CIAT, Cali, Colombia. 


\section{Referencias hemerográficas}

1. Autoridad Autónoma de Cuenca Hidrográfica Chira Piura y PDRS, GTZ (2004). Diagnóstico Participativo con el Enfoque de Gestión del Riesgo de la Cuenca del Río Piura, Piura, Perú

2. CEDEPAS NORTE y Proyecto Regional Cuencas Andinas (2007). Estudio Socio- económico y de los Sistemas de Producción en la Cuenca del Río Jequetepeque, Cajamarca, Perú.

3. Machim , M. (2006). Valoración económica de los recursos naturales: Perspectivas a través de los diferentes enfoques de mercado. En: Revista Futuros N. ${ }^{\circ} 13$ Vol IV.
4. Banco Interamericano de Desarrollo-BID (2005). Estudio sobre inversión directa en negocios forestales sostenibles - proyecto atn/ np-8323-r. Índice de atracción a la inversión forestal (IAIF) Informe Final - rev. 01. División de administración de Recursos Naturales y Medio Ambiente, Departamento Regional de Operaciones. Curitiba - Brasil. Abril / 2005.

\section{Referencias informáticas}

1. Vidal, S. Activos Ambientales, Visita 2-10-12 www.slideshare.net/wshy/anal - Colombia 